\title{
Vaccines to Tackle Antimicrobial Resistance
}

\author{
Ivana Haluskova Balter* \\ French Society of Immunology, France \\ *Corresponding Author: Ivana Haluskova Balter, French Society of Immunology, France.
}

Received: August 01, 2019; Published: October 16, 2019

DOI: 10.31080/ASMI.2019.02.0402

Bacteria, viruses, parasites and fungi that are resistant to drug cause 700,000 death each year. By 2050 superbugs inured to treatments could cause up to 10 million deaths annually and costs the global economy US\$100 trillion.

AMR (antimicrobial) resistance is regarded nowadays as a major threat to global public health. The issue is receiving high-level political attention (G7 and G20 in 2017 for first time). Pandemics, drug resistance and neglected diseases framing health as a "global security issue". The list was drawn up in a bid to guide and promote research and development ( $R$ and $D$ ) of new antibiotics, as part of WHO's efforts for AMR (27 $7^{\text {th }}$ Feb 2017) The World Health Organisation has launched the Global Antimicrobial Resistance Surveillance System (GLASS) initiative in order to strengthen bacteriology laboratories in low-and middle income countries for targeted antibiotic resistance surveillance.

Considering the threat that microbes and vectors resistance represent at the global scale it is necessary to bring together scientists, clinicians and public health experts to reinforce high-level research to identify, monitor or predict the emergence of resistant pathogens and vectors, highlight the current efforts in translational science and work to develop new tools and technologies necessary to tackle the challenges of diagnostic and surveillance of these health threats to examine innovative solutions to practically develop these tools on the field in resource-limited settings and emerging countries are essential players.

Tuberculosis (MDR/XDR) and latent tuberculosis represent a major issue to tackle attracts global attention as witnessed by recent WHO and inter-ministerial meeting in November 2017, high level UN meeting in NYC 2018, BRICs meeting in South Africa and many others.
Problem of resistance get worsened due declining number of new antibiotics and limited number of new classes. Multifaceted strategy to promote and prioritize highly potential alternatives to tackle AMR like vaccines development is required. Vaccines like diphtheria and tetanus did not prompt resistance. In 1980 the smallpox vaccine had eradicated the naturally circulating virus worldwide without generating resistance $[1,2]$.

Additionally, introduction of live vaccines like measles and BCG has been associated with much larger reduction of morality than can be explained by the prevention of the targeted infections and recent research like LATV pertussis [3] highlights importance of "off target" effects to be evaluated in depth.

Thoughtful and innovative research and development taking into account host microbiota "superorganism" and immune crosstalk - Immune system training open large avenue for future development including new antimicrobials with protective effect on microbiota. [4] and measures to diminish transfer of AR genes and prevent complications related to antibiotic use (dysbiosis, CDIs). Research shows that microbiome (microbiome mediated immune response) might be implicated into vaccine responsiveness. Accurate diagnostic and surveillance with better understanding of genetic and immunologic background of host specific response and pathogen evolution drives successful country adapted vaccine research.

Vaccines, as highly potent tool and valuable alternative from long term perspective being clearly recognized as a major tool for public health already. Their strength to avoid effect on microbiome and microbiome /immune mediated effect and known beneficial effect listed place them as really strong path to explore. 
Futher strong support to promote various paths like vaccines, phototherapy etc as highly potential alternatives to tackle antibiotic resistance need joint endorsement including regulatory and economic stakeholders along with necessary partnership and advocacy at Global level.

\section{Bibliography}

1. The review on antimicrobial resistance. Antimicrobial resistance/Tackling a crisis for the health and wealth of nation (WT)

2. UA/EM Tackling the antimicrobial resistance innovation crisis WHO Assembly

3. C Locht. On and off" target effects of LATV, French immunology congress (2017).

4. Microbes, Immunity and Metabolism, Institute Pasteur Paris (2017).

Volume 2 Issue 11 November 2019

(C) All rights are reserved by Ivana Haluskova Balter. 